\title{
Detectability of large-scale flows in global helioseismic data - A numerical experiment
}

\author{
M. Roth ${ }^{1, \star}$, R. Howe ${ }^{2}$, and R. Komm ${ }^{2}$ \\ 1 Kiepenheuer-Institut für Sonnenphysik, 79104 Freiburg, Germany \\ ${ }^{2}$ National Solar Observatory ${ }^{\star}$, 950 N. Cherry Ave., Tucson, AZ 85719, USA \\ Received 5 November 2001 / Accepted 18 September 2002
}

\begin{abstract}
Convective motions affect the solar $p$-modes by shifting their frequencies. In comparison to the frequency splitting caused by the differential rotation, this is only a small additional effect. As the spatial resolution of the inversions for the differential rotation becomes better, it is important to know how these additional frequency shifts modify the splitting coefficients and how these two effects might be disentangled. Therefore we carry out a numerical experiment. We use quasi-degenerate perturbation theory to create frequencies of $p$-modes that are affected by differential rotation and by large-scale flows. The simulated frequency sets are analyzed and inverted for differential rotation. We use changes in the $(l, v)$ coverage, the multiplets, and the inversion results as diagnostics to draw conclusions about the detectability of large-scale flows in global helioseismic data. The result is a detectability limit of the order of $10 \mathrm{~m} \mathrm{~s}^{-1}$ for large-scale flows in the convection zone. A sectoral poloidal flow with greater amplitude will lead to a noticeable distortion of the rotation rate, while a zonal poloidal flow with greater amplitude will lead to distorted even- $a$ coefficients and disrupted multiplets.
\end{abstract}

Key words. convection - Sun: rotation - Sun: oscillations

\section{Introduction}

In this study, we search for large-scale, long-lived structures in the convection zone by using global helioseismology results combined with quasi-degenerate perturbation theory and numerical simulations. We concentrate on changes in the $(l, v)$ coverage, the multiplets, and the inversion results and derive detectability limits for the velocity of large-scale structures.

The motivation for this study is two-fold. On the one hand, the internal structure of the Sun has been determined with increasing accuracy within the last few years. Especially with the availability of high-quality helioseismic data the properties of the differential rotation and its depth dependence have been studied in great detail by inverting oscillation data. Because of the improving resolution of the solar interior, it is necessary to know about smaller effects of physical processes in the sun on the solar oscillations and their influences on the inversion results.

One of these processes inside the sun is the possible effect of large-scale convection cells on the solar $p$-modes. It has been

Send offprint requests to: M. Roth,

e-mail: mroth@kis.uni-freiburg.de

* Present address: Freiburg Center for Data Analysis and Modelling, Eckerstr. 1, 79104 Freiburg, Germany.

$\star \star$ Operated by the Association of Universities for Research in Astronomy, Inc. under cooperative agreement with the National Science Foundation. shown by perturbation theory how to solve the forward problem to calculate the influence of toroidal and poloidal velocity fields on the oscillations (Lavely \& Ritzwoller 1992). The calculations that deal with the zonal toroidal flows representing the differential rotation have been used to develop inversion methods (Schou et al. 1998). But, so far, there exists no inversion method that based on global helioseismic data is able to determine large-scale poloidal or non-zonal toroidal flow components inside the convection zone. Therefore our investigation might be helpful in developing such inversion methods.

On the other hand the search for large-scale flows, other than solar rotation, in the convection zone has the potential to improve our understanding of the dynamics of the solar convection zone. The hypothesis that convective elements larger than supergranulation exist goes back about 40 years. Simon \& Weiss (1968) postulated according to mixing length theory that the largest possible convective pattern extends over the depth of the convection zone, the so-called giant cells. However, such a pattern has not been observed unambiguously despite an extensive search by several groups (for example, Schröter et al. 1978; LaBonte et al. 1981; Cram et al. 1983; Robillot et al. 1984; Bertello \& Restaino 1993). Recently, Beck et al. (1998) reported observing long-lived cells elongated in longitude with amplitudes of few m s${ }^{-1}$, while Ulrich (2001) interprets a similar pattern as higher-order components of the banded zonalflow pattern (also known as torsional oscillations). Hathaway et al. (2000) concluded that giant cells do not exist as a distinct mode of convection. Current global simulations of the 
convection zone (Elliott et al. 2000; Miesch et al. 2000) show large-scale flow patterns that are more complex than simple rolls or banana-shaped cells. Local helioseismology techniques, such as ring diagrams (for example, Haber et al. 2000), are presently limited to near surface layers and cannot be used to determine flows throughout the whole convection zone. Therefore our contribution can help to search for distinct modes of convection, to restrict the parameter space for the numerical simulations and to develop more powerful methods to determine flows within the deeper layers of the convection zone.

In our attempt to detect large-scale flows with global helioseismic data we concentrate on the application of quasidegenerate perturbation calculations. Following Lavely \& Ritzwoller (1992), Roth \& Stix (1999) show that poloidal flow components cause small additional frequency shifts compared to the influence of the differential rotation. In order to learn more about the strength of the effect of large-scale flows in the convection zone and their possible detectability, we will use quasi-degenerate perturbation theory to calculate the frequencies of oscillations that are influenced by both differential rotation and large-scale poloidal velocity fields. These artificial frequencies are inverted for differential rotation by the same RLS (Regularized Least Squares) inversion method used for inverting data from the Global Oscillation Network Group (GONG), for example by Howe et al. (2000). This allows us to derive an upper limit for the significance of the poloidal flow field, depending on its amplitude and geometry, which is of the order of $10 \mathrm{~m} \mathrm{~s}^{-1}$.

\section{Perturbation theory}

Our aim is to investigate smaller effects like slow streaming large-scale poloidal fields. The first step is to solve the forward problem, i.e., to calculate theoretically the influence on the solar acoustic modes. We will follow the work of Lavely \& Ritzwoller (1992). The starting point for these calculations is a reference model; we base our work on "Model S" from Christensen-Dalsgaard et al. (1996). The reference model describes the Sun as a star in hydrostatic equilibrium. It is one-dimensional, i.e., all physical quantities depend on one variable, the distance $r$ from the solar center. It follows that the solar model is spherically symmetric, non-rotating, nonmagnetic, and static.

This reference model is subject to acoustic modes, which are characteristic spatial displacement patterns $\delta \boldsymbol{r}(r, \theta, \phi ; \tau)$, that oscillate with fixed frequencies $(\theta$ and $\phi$ are the colatitude and the longitude; $\tau$ is the time). The equation of motion of such an oscillatory displacement $\delta \boldsymbol{r}=\boldsymbol{\xi} \exp \{-\mathrm{i} \omega \tau\}$ with amplitude $\xi(r, \theta, \phi)$ and angular frequency $\omega$ is derived in Christensen-Dalsgaard (1998) and is given by

$\rho_{0} \frac{\partial^{2} \boldsymbol{\delta} \boldsymbol{r}}{\partial \tau^{2}}=-\omega^{2} \rho_{0} \delta \boldsymbol{r}=-\nabla p^{\prime}+\rho_{0} \boldsymbol{g}^{\prime}+\rho^{\prime} \boldsymbol{g}_{0}$

where $p^{\prime}, \rho^{\prime}$, and $\boldsymbol{g}^{\prime}$ are perturbations of the equilibrium quantities pressure $p_{0}$, density $\rho_{0}$ and gravitational acceleration $\boldsymbol{g}$.

Equation (1) has a spectrum of eigensolutions with eigenvalues $\omega_{k}^{2}$, where $k=(n, l, m)$ stands for a triplet of indices, respectively, the radial order, the harmonic degree, and the

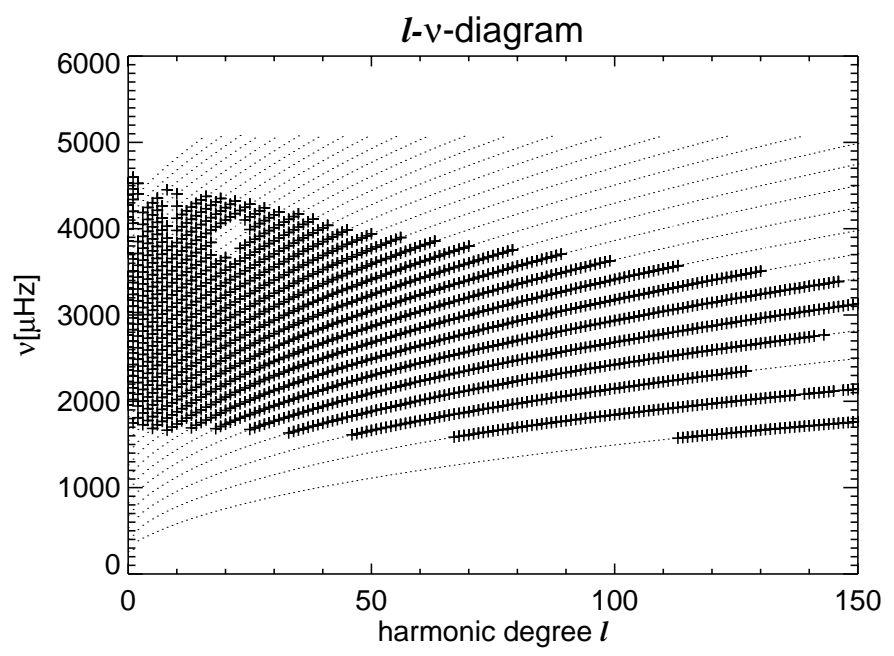

Fig. 1. $(l, v)$ diagram for the used solar model. The marked modes are the ones used for the numerical experiment and correspond to the modes observed by GONG.

azimuthal order. We will concentrate on the $p$-modes whose restoring force is the pressure gradient.

For a spherical symmetric solar model the solar $p$-modes are degenerate, i.e., each $p$-mode of a multiplet that consists of the $2 l+1$ eigenfunctions with identical $n$ and $l$ values has the same frequency $\omega_{n l}$ independent of $m$. In Fig. 1 we show the spectrum or $(l, v)$ diagram of the $p$-modes with $l \leq 150$ and $v=\omega / 2 \pi \leq 5 \mathrm{mHz}$ that were calculated on the basis of "Model S" (Christensen-Dalsgaard et al. 1996).

In this paper we investigate velocity fields $v$ in the convection zone; they lift the degeneracy by an additional advection term in the equation of motion (1), (Christensen-Dalsgaard 1998; Lavely \& Ritzwoller 1992)

$-\omega^{2} \rho_{0} \boldsymbol{\xi}-2 \mathrm{i} \omega \rho_{0}(\boldsymbol{v} \cdot \nabla) \boldsymbol{\xi}=-\nabla p^{\prime}+\rho_{0} \boldsymbol{g}^{\prime}+\rho^{\prime} \boldsymbol{g}_{0}$.

If the magnitude of the velocity $v$ is small compared with the sound speed, first-order perturbation theory can be used to calculate corrections $\delta \omega^{2}$ of the eigenvalue $\omega^{2}$ caused by the influences of velocity fields

$\delta \omega^{2}=-2 \mathrm{i} \omega \frac{\int \rho_{0} \boldsymbol{\xi}_{k^{\prime}}^{*} \cdot(\boldsymbol{v} \cdot \nabla) \boldsymbol{\xi}_{k} \mathrm{~d}^{3} r}{\int \rho_{0}\left|\boldsymbol{\xi}_{k^{\prime}}\right|^{2} \mathrm{~d}^{3} r}$

Here we treated the Hermitian operator

$\mathcal{H}_{1} \equiv-2 \mathrm{i} \omega \rho_{0}(\boldsymbol{v} \cdot \nabla)$

in Eq. (2) as perturbation of the Hermitian operation

$\mathcal{H}_{0} \boldsymbol{\xi} \equiv-\nabla p^{\prime}+\rho_{0} \boldsymbol{g}^{\prime}+\rho^{\prime} \boldsymbol{g}_{0}=-\omega^{2} \rho_{0} \boldsymbol{\xi}$.

For our purposes $v$ is decomposed into a toroidal and a poloidal field and the two components are expanded in spherical harmonics $Y_{s}^{t}$ defined by

$$
\begin{aligned}
Y_{s}^{t}(\theta, \phi)= & \frac{(-1)^{s+t}}{2^{s} s !}\left[\frac{(2 s+1)(s-t) !}{4 \pi(s+t) !}\right]^{1 / 2} \\
& \times(\sin \theta)^{t}\left[\frac{\partial}{\partial(\cos \theta)}\right]^{s+t}(\sin \theta)^{2 s} \mathrm{e}^{\mathrm{i} t \phi}
\end{aligned}
$$


with harmonic degree $s$ and azimuthal order $t$. Hence

$\boldsymbol{v}(\boldsymbol{r})=\sum_{s=0}^{\infty} \sum_{t=-s}^{s}\left[\boldsymbol{T}_{s}^{t}(r, \theta, \phi)+\boldsymbol{P}_{s}^{t}(r, \theta, \phi)\right]$,

where

$\boldsymbol{T}_{s}^{t}(r, \theta, \phi)=-w_{s}^{t}(r) \boldsymbol{e}_{r} \times \nabla_{h} Y_{s}^{t}(\theta, \phi)$

is the toroidal and

$\boldsymbol{P}_{s}^{t}(r, \theta, \phi)=u_{s}^{t}(r) Y_{s}^{t}(\theta, \phi) \boldsymbol{e}_{r}+v_{s}^{t}(r) \nabla_{h} Y_{s}^{t}(\theta, \phi)$,

is the poloidal field component. The quantities $w_{s}^{t}(r), u_{s}^{t}(r)$, and $v_{s}^{t}(r)$ are the depth-dependent expansion coefficients, and $\nabla_{h}$ is the surface gradient operator (Lavely \& Ritzwoller 1992). This allows us to study the influence of differential rotation and of convection cells on the mode frequencies.

\subsection{Differential rotation}

The differential rotation, which is a pure toroidal velocity field, can be expanded in zonal components of spherical harmonics $Y_{s}^{0}$ according to Eqs. (7) and (8)

$\boldsymbol{v}_{\mathrm{rot}}(\boldsymbol{r})=\sum_{s}-w_{s}^{0}(r) \boldsymbol{e}_{r} \times \nabla_{h} Y_{s}^{0}(\theta)$.

The results of a degenerate perturbation calculation $\left(n^{\prime}=n\right.$, $l^{\prime}=l$ in Eq. (3)) are corrected frequencies that depend on the azimuthal order $m$

$$
\begin{aligned}
\omega_{n l}(m) & =\omega_{n l}+\sum_{s \text { odd }} \int_{0}^{R} K_{n l s}(r) w_{s}^{0}(r) r^{2} \mathrm{~d} r\left(\begin{array}{ccc}
s & l & l \\
0 & m & -m
\end{array}\right) \\
& =\omega_{n l}+\sum_{s \text { odd }} a_{s}(n, l) P_{s}^{(l)}(m),
\end{aligned}
$$

where $K_{n l s}(r)$ depends on the eigenfunction and the geometry of the velocity field component indexed with $s$. The last factor in Eq. (11) is a Wigner 3j symbol (Edmonds 1960), for which we also use the notation $\left(l_{1} l_{2} l_{3} / m_{1} m_{2} m_{3}\right)$, with the $l_{i}$ as the harmonic degrees and the $m_{i}$ as the azimuthal orders. The sum in Eq. (11) is only carried out over odd $s$ because $K_{n l s}(r)$ vanishes for even $s$ (Lavely \& Ritzwoller 1992). The frequency shift can also be expressed more generally in terms of any orthogonal functions $P_{s}^{(l)}(m)$. This is given in Eq. (12), where we imply that symmetry properties of these functions lead to a mirroring of the rotation in the odd components. Hence, the inferred rotation rates are symmetric about the equator. The expansion coefficients $a_{s}(n, l)$ are weighted averages of the component $w_{s}^{0}(r)$ of the rotation

$a_{s}(n, l)=\int_{0}^{R} K_{n l s}(r) w_{s}^{0}(r) r^{2} \mathrm{~d} r$.

The weighting is governed by the kernels $K_{n l s}(r)$ that depend on the region a mode samples (moreover the kernels depend on the structure of the solar interior, so that the determination of the frequency shift and later on the inversion for the solar interior are only as good as the model used). Hence the differential

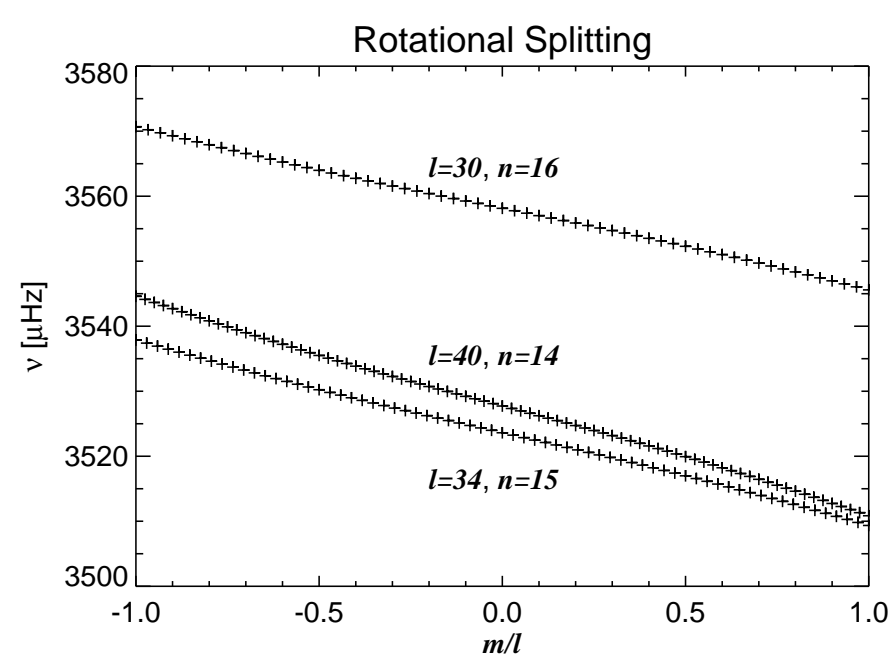

Fig. 2. Lifting of degeneracy by differential rotation ( $\operatorname{solar} \Omega(r, \theta)$, first obtained by inversion of GONG data) for several multiplets $l=34$, $n=15 ; l=40, n=14 ; l=30, n=16$.

rotation results in a lifting of the degeneracy of the $p$-mode frequencies. Figure 2 shows an example for three multiplets (we use the same sign convention as GONG where within a multiplet frequencies decrease with increasing $m$ as adopted by Hathaway 1992).

\subsection{Large-scale flows}

In this section we study the effect of large-scale flows, located in the convection zone, on solar oscillations. By large-scale flows we mean flows that have only poloidal and non-zonal toroidal components. We refer to zonal toroidal components as differential rotation, for which we already did the calculations in the last section.

We focus only on the effect of the poloidal components on the solar oscillations, because the effect of the non-zonal toroidal flow components should be the smallest (Roth 2001). A poloidal flow field will result in a frequency shift of $\delta \omega=0$ in the case $\left(n^{\prime}, l^{\prime}, m^{\prime}\right)=(n, l, m)$, because of three selection rules (Lavely \& Ritzwoller 1992). The first two rules arise from properties of the Wigner $3 \mathrm{j}$ symbols which vanish except when the harmonic degrees $l, l^{\prime}$, and $s$ satisfy a triangular condition (Edmonds 1960), i.e.

$\left|l^{\prime}-s\right| \leq l,|s-l| \leq l^{\prime},\left|l-l^{\prime}\right| \leq s$,

and the azimuthal orders satisfy the equation

$t+m-m^{\prime}=0$

The third rule comes from the radial integration in (3) and means that toroidal flows contribute to the frequency shift only if $s+l+l^{\prime}$ is odd, and poloidal flows only if $s+l+l^{\prime}$ is even. In the case of self-coupling, i.e. $\left(n^{\prime}, l^{\prime}\right)=(n, l)$, poloidal flows have no influence on the frequencies. But for $\left(n^{\prime}, l^{\prime}\right) \neq(n, l)$ the calculations of quasi-degenerate perturbation theory result in nonzero frequency shifts. Because of the analogy to the coupling of angular momenta this is called coupling of p-modes. For the 
coupling of only two modes the shifted squares of the frequen$\operatorname{cies} \omega_{n l}^{2}$ and $\omega_{n^{\prime} l^{\prime}}^{2}$ of the two $p$-modes involved are given by

$$
\begin{aligned}
\omega_{n l / n^{\prime} l^{\prime}}^{2}(m)= & \frac{\omega_{n l}^{2}+\omega_{n^{\prime} l^{\prime}}^{2}}{2} \\
& \pm \frac{1}{2} \sqrt{\left(\omega_{n l}^{2}-\omega_{n^{\prime} l^{\prime}}^{2}\right)^{2}+4\left|H_{n n^{\prime} l l^{\prime}}^{m m^{\prime}}\right|^{2}}
\end{aligned}
$$

where

$$
H_{n n^{\prime} l l^{\prime}}^{m m^{\prime}}=\int_{0}^{R} K_{n n^{\prime} l l^{\prime} s}(r) u_{s}^{t}(r) r^{2} \mathrm{~d} r\left(\begin{array}{ccc}
s & l & l^{\prime} \\
t & m & -m^{\prime}
\end{array}\right)
$$

with $K_{n n^{\prime} l l^{\prime} s}(r)$ being a kernel that depends on the eigenfunctions of the modes involved in the coupling and the angular geometry of the flow field (for a derivation of $K_{n n^{\prime} l l^{\prime} s}(r)$ compare with Eq. (90) in Lavely \& Ritzwoller 1992). This means that coupling $\left(H_{n n^{\prime} l l^{\prime}}^{m m^{\prime}} \neq 0\right)$ causes the frequency of one partner to be shifted upwards and that of the other partner shifted downwards (Roth \& Stix 1999). As $t$ is not necessarily equal to zero, the shifts within a multiplet are, due to properties of the Wigner $3 \mathrm{j}$ symbols, not necessarily symmetric about the frequency of the mode with azimuthal order $m=0$, which may itself be shifted. Coupling of one mode with several other modes is also possible; in this case the shifted frequencies are determined from the eigenvalues of the supermatrix $Z$ with elements $Z_{n n^{\prime} l l^{\prime}}^{m m^{\prime}}=\omega_{n l}^{2} \delta_{n n^{\prime}} \delta_{l l^{\prime}} \delta_{m m^{\prime}}+H_{n n^{\prime} l l^{\prime}}^{m m^{\prime}}$ described in Lavely \& Ritzwoller (1992). This shows that the supermatrix $Z$ is the sum of two matrices, a diagonal matrix consisting of $(2 l+1) \times(2 l+1)$ block matrices with entries $\omega_{n l}^{2}$ and the coupling matrix consisting of $(2 l+1) \times\left(2 l^{\prime}+1\right)$ block matrices with the elements $H_{n n^{\prime}, l l^{\prime}}^{m m^{\prime}}$. The azimuthal-order superscripts determine the position of the matrix element within the respective block matrix.

For the numerical determination of the shifted frequencies it is helpful to limit the number of possible coupling partners by the requirement $\left|\omega_{n^{\prime} l^{\prime}}-\omega_{n l}\right| \leq \Omega_{\text {range }}$ with given $\Omega_{\text {range }}$. In principle, we have to include all possible coupling partners. But according to Eq. (16), the strength of the coupling decreases with increasing difference in frequency, as can be seen by an expansion of the square root. This is characteristic for perturbation theory of quasi-degenerate eigenstates; it is for this reason that only modes with frequencies in close vicinity need to be taken into account. For the highest amplitudes of the velocity fields used in our calculations the contributions become more and more negligible for frequency differences above $\Omega_{\text {range }}^{\max }=30 \mu \mathrm{Hz}$. We can thus limit the number of possible partners and speed up the numerical calculations by setting $\Omega_{\text {range }}=2 \Omega_{\text {range }}^{\max }$ without loss of generality. Moreover the selection rules will further reduce the number of couplers.

Coupling of modes leads to additional, and in the case $t \neq 0$ to asymmetric, shifts in a multiplet, cf. the examples in Figs. 3 and 4 .

At this point we add a few remarks about the symmetry of the Wigner $3 \mathrm{j}$ symbols and the resulting sensitivity to various flows. These remarks will become essential for the understanding of the results presented in Sect. 4. The differential rotation leads to splittings that are anti-symmetric with respect to $m=0$ (cf. Fig. 2); the Wigner 3j symbols that represent these splitting are the $(s l l / 0 m-m)$-symbols. Due to the third selection



Fig. 3. Asymmetric shifted frequencies within the multiplet $l=34$, $n=15$ due to coupling to modes from the multiplets $l=40, n=14$ and $l=30, n=16$, caused by a poloidal flow field $s=8, t=8$ with $u_{\text {char }}=100 \mathrm{~m} \mathrm{~s}^{-1}$. The arrows indicate which shifts are caused by which coupling partner.



Fig. 4. The multiplet $l=34, n=15$ and its coupling partners affected by a zonal poloidal flow $s=8, t=0, u_{\text {char }}=100 \mathrm{~m} \mathrm{~s}^{-1}$. The resulting frequency shifts are symmetric to $m=0$.

rule the contribution to the frequency shift is non-zero only for odd $s$. Therefore the expansion of the rotational splitting was given by the odd $a_{s}$-coefficients. Conversely the meridional flows lead to splittings symmetric about $m=0$ (cf. Fig. 4); the corresponding Wigner $3 \mathrm{j}$ symbols are the $\left(s l^{\prime} / 0 m-m\right)$ symbols. Due to the selection rules the contribution to the frequency shift is non-zero for even $s+l+l^{\prime}$. Therefore the 


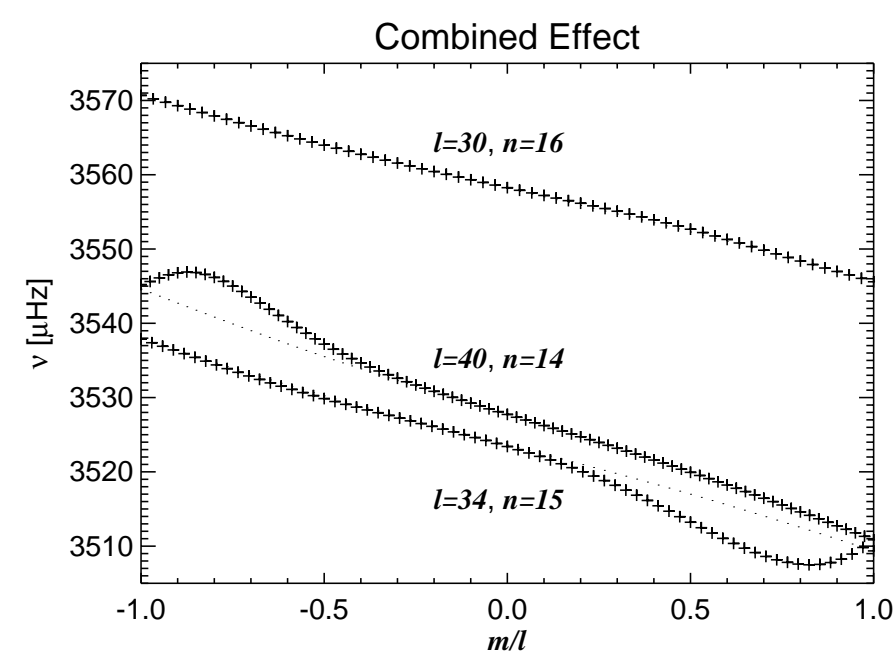

Fig. 5. Poloidal velocity fields (here $s=t=8$ ) lead to a deviation (+-symbols) from the rotational splitting (dots). To emphasize the deviation an amplitude $u_{\text {char }}=200 \mathrm{~m} \mathrm{~s}^{-1}$ was chosen.

expansion of the meridional-flow-splitting in terms of functions that are symmetric and anti-symmetric with respect to $m=0$ will be given by the expansion coefficients corresponding to the even functions. Any other poloidal flow field with $t \neq 0$ will result in a distribution of the frequency shifts with no symmetry with respect to $m=0$, due to the second selection rule (15); therefore these shifts cannot be assigned to only even or only odd functions in an expansion.

As result of the combined effect of differential rotation and poloidal velocity fields, the frequencies within a multiplet might be distributed as shown in the example of Fig. 5 for differential rotation plus a poloidal velocity field with $s=t=8$. Details on the models for the differential rotation and the poloidal flows underlying Figs. 2-5 are given in Sect. 3.1.

\section{Numerical experiment}

As a second step in our approach to gain knowledge about the small effect of velocity fields on the solar oscillations we will use perturbation theory to carry out a numerical experiment.

We create artificial oscillation frequencies, that cover the whole $(l, v)$ diagram in a range $0 \leq l \leq 150$ for the harmonic degree and all respective azimuthal orders. As the availability of modes is restricted to the observation we also use only those multiplets which are observed, cf. Fig. 1. Finally these artificial frequencies are inverted for differential rotation.

\subsection{Simulated oscillation data}

The process of creating the data is the following: the frequencies are determined as eigenfrequencies of a standard solar model. We use the "Model S" from J. Christensen-Dalsgaard to determine the degenerate oscillations.

Afterwards we perform a perturbation calculation to take the influence of differential rotation into account. As an input model for the differential rotation we use an inversion result, that is an average of six three-month periods of GONG data, beginning in March 1996 and ending in January 1998 (GONG

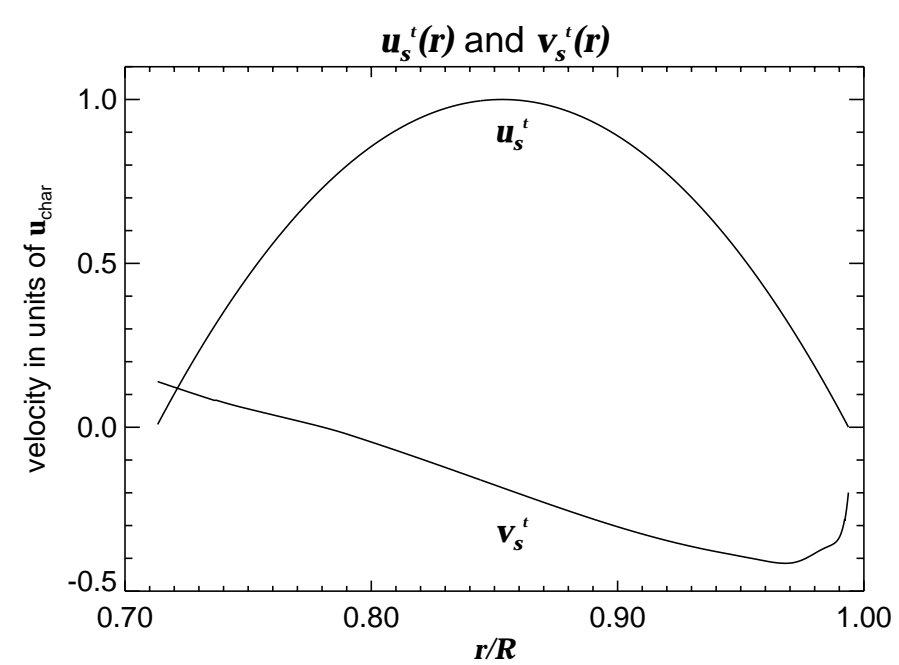

Fig. 6. The depth-dependent expansion coefficients $u_{s}^{t}(r)$ and $v_{s}^{t}(r)$ of the radial and horizontal component of the poloidal velocity fields used $(s=8)$.

months 10-27). The profile is similar to that shown in Howe et al. (2000). The result is the splitting of the degenerate oscillations into frequency multiplets, i.e. the frequency has now become a function of the azimuthal order $m$ (Fig. 2).

In the next step we add one component $\boldsymbol{P}_{s}^{t}(\boldsymbol{r})$ of the poloidal flow field and use quasi-degenerate perturbation theory. The model for the flow component $\boldsymbol{P}_{s}^{t}(\boldsymbol{r})$ was given by Eq. (9). We assume that $\mathrm{d} \boldsymbol{P}_{s}^{t} / \mathrm{d} \tau=0$ and hence $\nabla \cdot\left(\rho_{0}(r) \boldsymbol{P}_{s}^{t}\right)=0$ so that $v_{s}^{t}(r)$ can be expressed in terms of $u_{s}^{t}(r)$

$v_{s}^{t}(r)=\frac{\partial_{r}\left(r^{2} \rho_{0} u_{s}^{t}(r)\right)}{\rho_{0} r s(s+1)}$.

The quantity $u_{s}^{t}(r)$ is taken to be

$u_{s}^{t}(r)=u_{\mathrm{char}} \frac{4(R-r)\left(r-r_{\mathrm{conv}}\right)}{\left(R-r_{\mathrm{conv}}\right)^{2}}$ for $r_{\mathrm{conv}} \leq r \leq R$,

where $u_{\text {char }}$ is a characteristic parameter that allows us to control the strength of the flow field component, $R$ and $r_{\text {conv }}$ are the solar radius and the bottom of the convection zone. The resulting depth dependence of $u_{s}^{t}(r)$ and $v_{s}^{t}(r)$ is shown in Fig. 6. The radial component $u_{s}^{t}(r)$ of the flow vanishes at the top and the bottom of the convection zone and has its maximum value $u_{\text {char }}$ in the middle of the convection zone. In between we have chosen a simple parabolic dependence of $u_{s}^{t}$ on the radius $r$.

The horizontal expansion coefficient $v_{s}^{t}(r)$ changes its sign, so that a laminar flow on closed streamlines is established. The difference in amplitude of $v_{s}^{t}$ between top and bottom of the convection zone reflects the density stratification. At the top the amplitude is large because of the low density, at the base of the convection zone the density is higher, therefore only a small amplitude in $v_{s}^{t}$ is necessary to conserve the mass flow.

In general such poloidal velocity fields have the properties of Bénard convection, i.e. cellular flows with upwards streaming matter in the center and downward streams at the borders of the cells. The geometry and the amplitude of the flow field is fixed by the parameters $s, t$, and $u_{\text {char }}$. We discuss in Sect. 4 the results for the two extreme cases $s=t$ (sectoral cells) and 

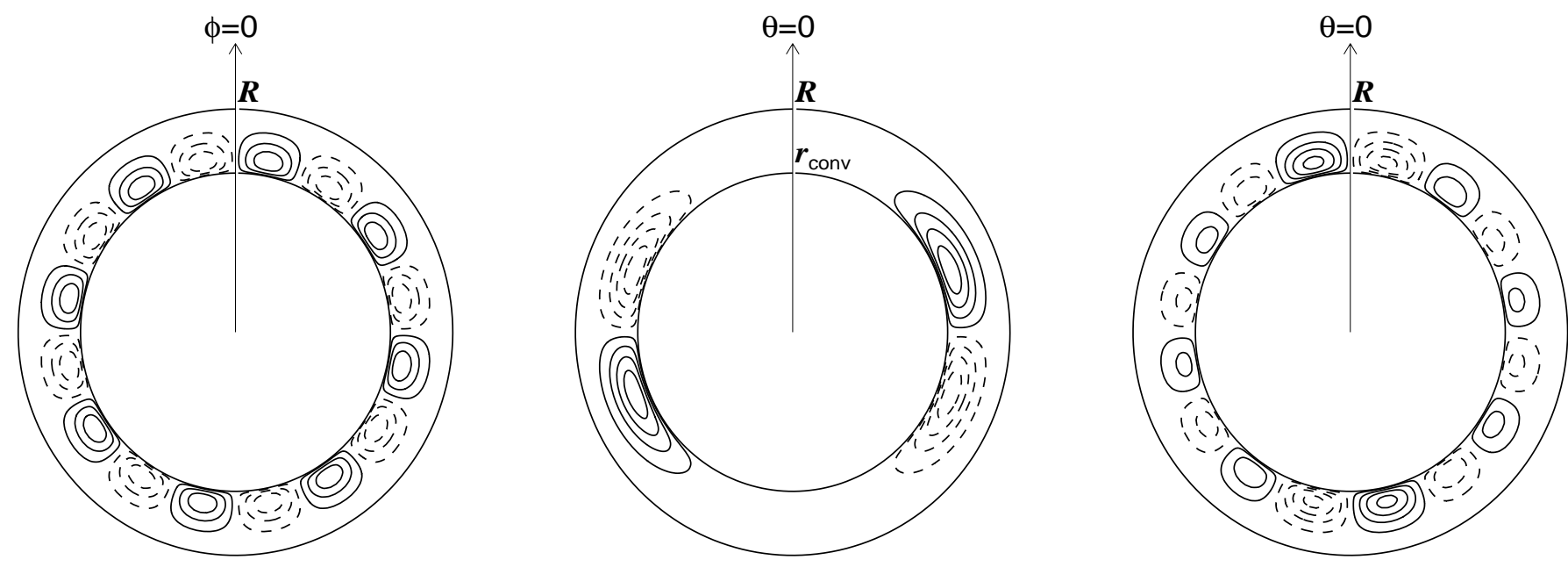

Fig. 7. Streamlines of the equatorial (left) and meridional ( $\phi=0$, middle) sections through the poloidal flow field $s=8, t=8$, and the meridional section through the poloidal flow field $s=8, t=0$ (right). The counterclockwise flows are marked with solid lines, the clockwise flows with dashed lines.

$t=0$ (zonal cells). A visualization of such field components is given in Fig. 7. The plots on the left and in the middle show streamlines of an equatorial and a meridional section through the poloidal flow field $s=8, t=8$, and the right plot shows the meridional section through the poloidal flow field $s=8, t=0$. Since a poloidal field with $t=0$ does not have components in direction of the rotation, an equatorial section through the flow is not useful to plot.

\subsection{Inversion for angular velocity}

After generating the frequencies we use these numerically determined frequencies as helioseismic data and invert them for differential rotation. For this purpose the frequencies within a multiplet, i.e., frequencies of oscillations with identical $n$ and $l$, are parameterized in terms of the central frequency $\omega_{n l}$ and less than $2 l+1$ coefficients $a_{s}$ such that

$\omega_{n l}(m)=\omega_{n l}+\sum_{s=1}^{s_{\max }} a_{s}(n, l) P_{s}^{(l)}(m)$.

The basis functions are orthogonal polynomials, which are related to Clebsch-Gordon coefficients $C_{s 0 l m}^{l m}$ (Ritzwoller \& Lavely 1991) by

$P_{s}^{(l)}(m)=(-1)^{s} \sqrt{\frac{2 s+1}{2 l+1}} C_{s 0 l m}^{l m}$

The Wigner $3 \mathrm{j}$ symbols that we used in Eqs. (11) and (17) are related to the Clebsch-Gordon coefficients by

$\left(\begin{array}{ccc}l_{1} & l_{2} & l_{3} \\ m_{1} & m_{2} & m_{3}\end{array}\right)=\frac{(-1)^{l_{1}-l_{2}+m_{3}}}{\left(2 l_{3}+1\right)^{1 / 2}} C_{l_{1} m_{1} l_{2} m_{2}}^{l_{3} m_{3}}$.

We are interested in the rotation rate. This is independent of the central frequency $\omega_{n l}$ and hence it does not matter whether our numerical model is able to reproduce frequencies that agree with the observed frequencies. This would be important if we were interested in a model of the Sun or the excitation of acoustic modes. The coefficients are combinations of the splittings and independent of $\omega_{n l}$.
In mathematical terms the rotation rate $\bar{\Omega}\left(r_{0}, \theta_{0}\right)$ at a given radius $r_{0}$ and a colatitude $\theta_{0}$ can be written as

$\bar{\Omega}\left(r_{0}, \theta_{0}\right)=\sum_{i=1}^{M} c_{i}\left(r_{0}, \theta_{0}\right) d_{i}$

where $d_{i}$ are the $M$ data values and where we assume that they are linear functionals of the true underlying rotation rate $\Omega(r, \theta)$ only

$d_{i}=\int_{0}^{R} \int_{0}^{\pi} K_{i}(r, \theta) \Omega(r, \theta) \mathrm{d} r \mathrm{~d} \theta$.

The kernels $K_{i}$ are presumed to be known functions. The $c_{i}$ are appropriately chosen coefficients. Equations (23) and (24) yield

$$
\begin{aligned}
\bar{\Omega}\left(r_{0}, \theta_{0}\right) & =\int_{0}^{R} \int_{0}^{\pi} \sum_{i=1}^{M} c_{i}\left(r_{0}, \theta_{0}\right) K_{i}(r, \theta) \Omega(r, \theta) \mathrm{d} r \mathrm{~d} \theta \\
& \equiv \int_{0}^{R} \int_{0}^{\pi} \mathcal{K}\left(r_{0}, \theta_{0} ; r, \theta\right) \Omega(r, \theta) \mathrm{d} r \mathrm{~d} \theta .
\end{aligned}
$$

The "averaging kernel" $\mathcal{K}$ for a given location $\left(r_{0}, \theta_{0}\right)$ describes the weighting of the true rotation rate to give an estimate of the inversion solution for that point. A full set of such averaging kernels would therefore cover the solar interior completely and describe the inverted rotation rate by an approximation $\bar{\Omega}(r, \theta)$.

Several inversion techniques have been developed. There exist two major families, the one is known as "Regularized Least Squares" or RLS (Tikhonov \& Arsenin 1977; Craig \& Brown 1986), the other as "Optimally Localized Average" (OLA; Backus \& Gilbert 1968). The inversion of Eq. (13) was carried out with a two-dimensional RLS fitting, which is described by Schou et al. (1998).

\section{Results}

In this section we present the result of the numerical experiment. We will concentrate on the flows with the geometries 
Sectoral Field
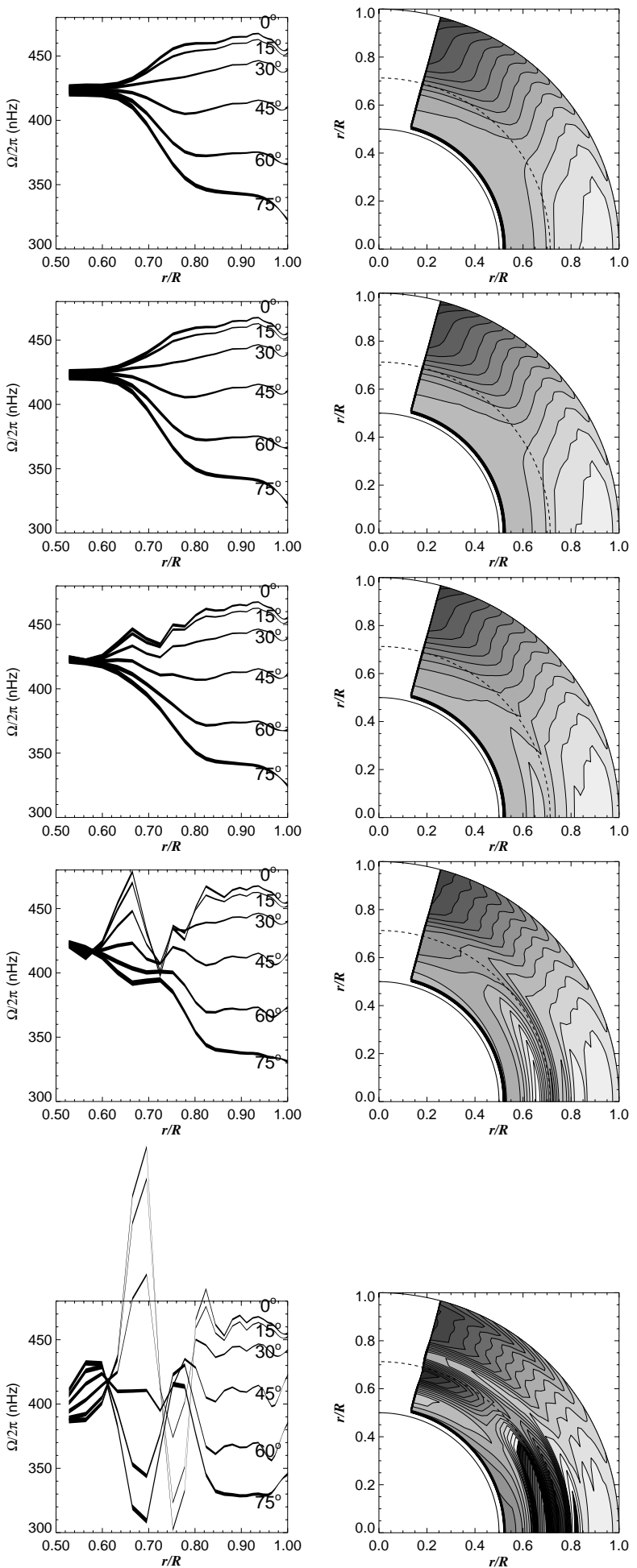

$u_{\text {char }}$

Zonal Field
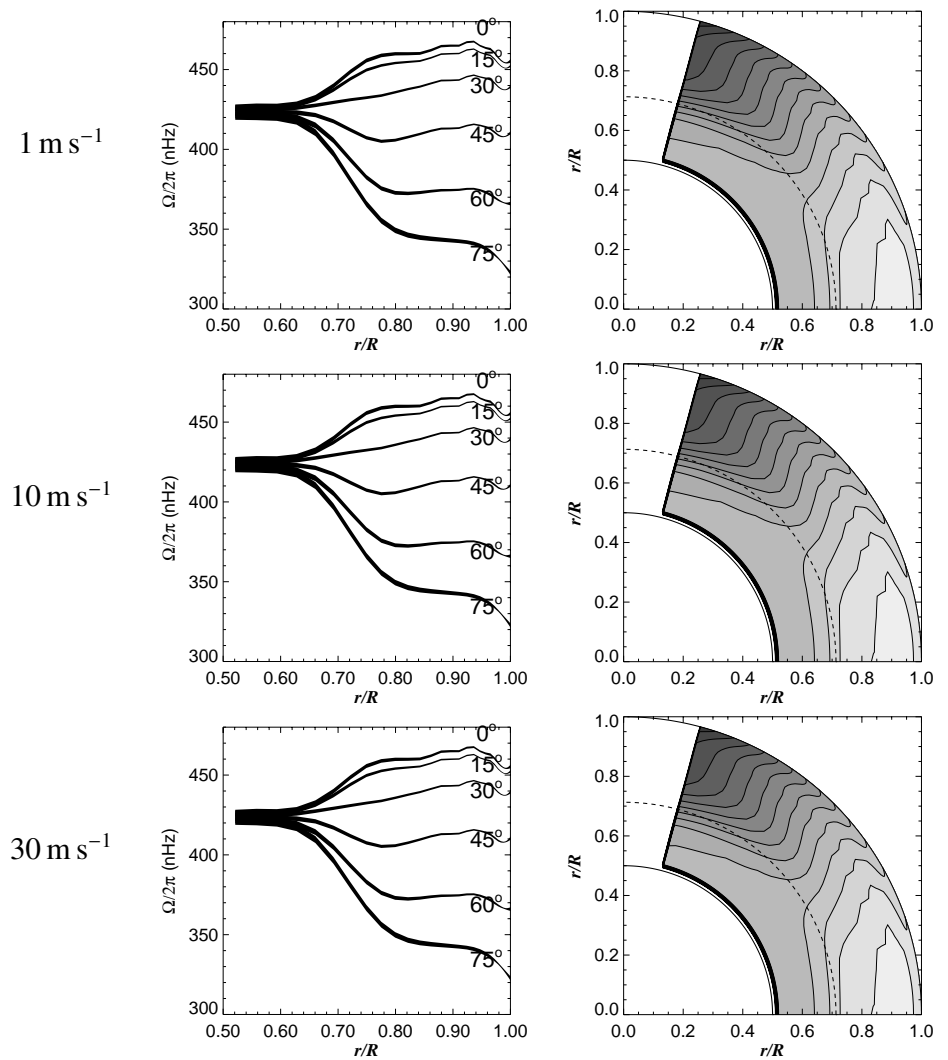

$60 \mathrm{~m} \mathrm{~s}^{-1}$
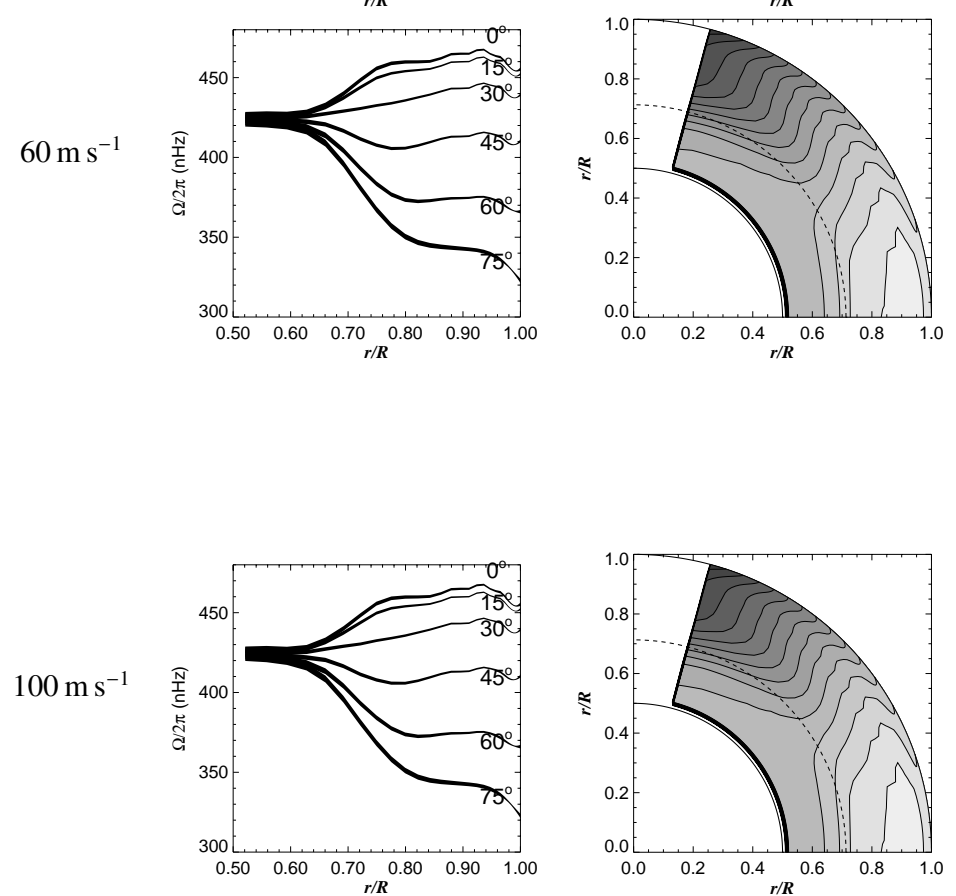

Fig. 8. The investigated inversions for poloidal flow fields $s=8$ with azimuthal order $t=8$ (sectoral cells, left columns) and $t=0$ (zonal cells, right columns). The respective characteristic parameters, $u_{\text {char }}$, are given in the middle column. The inversion is sensitive to the sectoral cells (strong dependence on the velocity amplitude), but insensitive to zonal cells.

$s=8, t=8$ (sectoral cells or banana cells) and $s=8$, $t=0$ (zonal cells or meridional rolls) both with characteristic parameters $u_{\text {char }}=1, \ldots, 100 \mathrm{~m} \mathrm{~s}^{-1}$ (strength of the flow field, defined in Eq. (19)). We also did calculations with flow fields with geometries $s=6, t=0, \ldots, 6 ; s=8, t=$ $0, \ldots, 8 ; s=10 ; t=0, \ldots, 10$ and characteristic parameters $u_{\text {char }}=1, \ldots, 500 \mathrm{~m} \mathrm{~s}^{-1}$, but all the inversions show similar results and are best discussed in terms of the chosen cases. 
Figure 8 displays the inversion results for flow fields with degree $s=8$ and orders $t=8$ (sectoral cells or banana cells) and $t=0$ (zonal cells or meridional rolls). The characteristic parameter $u_{\text {char }}$ increases from top to bottom from $1 \mathrm{~m} \mathrm{~s}^{-1}$ to $100 \mathrm{~m} \mathrm{~s}^{-1}$.

We note that the maximal velocity of the flow in the radial direction is in general not identical with $u_{\text {char }}$, because the maximum amplitude of the spherical harmonics given in Eq. (2) for our calculated cases is close to but not necessarily equal to one. The true maximal amplitude of $u_{s}^{t}(r)$ depends on $u_{\text {char }}, s$ and $t$. It is $0.51 u_{\text {char }}$ for the example $s=t=8$ and $1.16 u_{\text {char }}$ for $s=8, t=0$. For these examples the locations in the solar model where the maximal amplitude is reached can be identified in the left and right plots of Fig. 7 in the middle of the convection zone, at longitudes that are multiples of $\pi / 8$ for $t=8$ and at the poles for $t=0$. The corresponding normalization factors for the other geometries analyzed are in the same range. Therefore, the detectability limits derived in this section are valid for them as well.

\subsection{Sectoral cellular flows}

The influence of the additional poloidal field that is placed in the convection zone becomes noticeable at amplitudes higher than $u_{\text {char }}=10 \mathrm{~m} \mathrm{~s}^{-1}$ for the sectoral cells (Fig. 8, left two columns). As the flow field in direction of rotation is given by the $\phi$-component of the vector $\boldsymbol{P}_{s}^{t}$

$P_{s \phi}^{t} \propto v_{s}^{t} Y_{s}^{t}(\theta, \phi)$

the low latitudes are supposed to be affected more than the high latitudes. This is exactly what the inversion shows, the response decreases with increasing latitude. The plot with $u_{\text {char }}=$ $30 \mathrm{~m} \mathrm{~s}^{-1}$ shows that the low latitudes are already strongly distorted, while the high latitudes show the usual inversion results.

With further increasing amplitude the inversion shows that the higher latitudes also become affected. Moreover, artifacts below the convection zone become apparent, where a rigid body rotation was the input flow field. These artifacts are e.g., a deeper lying tachocline, and regions of alternating retarded and accelerated differential rotation. We will give an explanation for these artifacts later in Sect. 4.3.

\subsection{Zonal meridional flows}

For the zonal meridional flow field the inversions themselves do not show any influence of the poloidal convection cells (Fig. 8, right two columns). This is what we expect, because the zonal field does not have flow components in direction of the rotation. Thus, it gives confidence in the numerical simulations and the analysis techniques. But focusing on other quantities that are helpful like the expansion coefficients $a_{s}$ or the $(m, v)$ diagrams of the multiplets, the additional velocity field can be noticed. Concerning the $a_{s}$ the meridional flows are only reflected in the even coefficients; for the latter an increasing amplitude leads to an increasingly strong distortion of the multiplet. With large flows the structure of a multiplet can be disrupted to a degree that is not observed, cf. Fig. 9, where

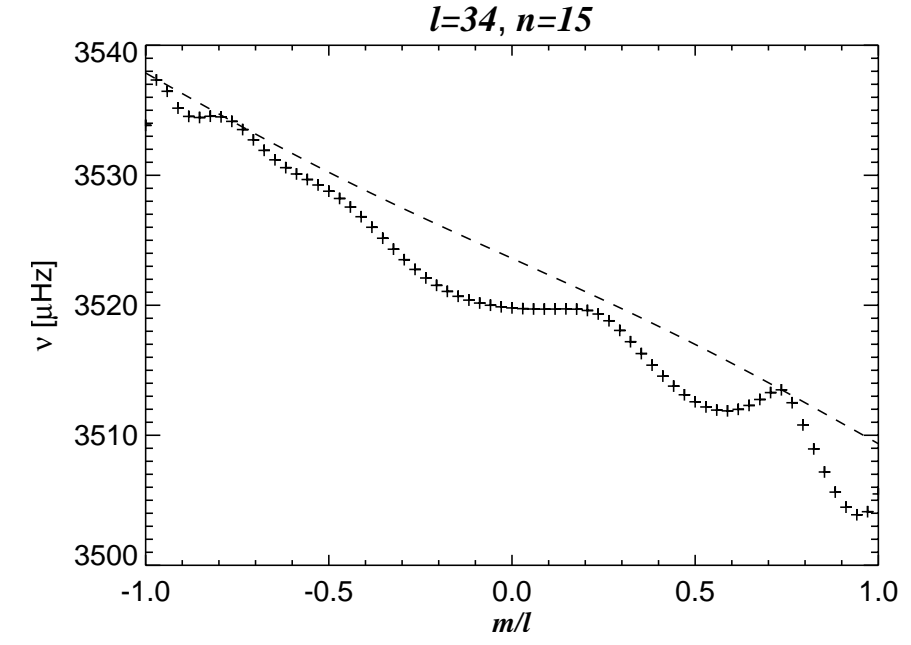

Fig. 9. Disrupted multiplet $l=34, n=15$ for differential rotation plus a poloidal flow $s=8, t=4, u_{\text {char }}=500 \mathrm{~m} \mathrm{~s}^{-1}$. Dashed line: undisturbed multiplet from Fig. 2.

we used a rather unrealistic amplitude of $500 \mathrm{~m} \mathrm{~s}^{-1}$ to emphasize the distortion of the multiplet.

Each point in a $(l, v)$ diagram represents a multiplet; if a multiplet is strongly distorted it cannot be fitted by only a few orthogonal polynomials (Eq. (20)). If that happens the whole multiplet is not taken into account for the inversion. This leads to pronounced gaps in the $(l, v)$ diagram for $u_{\text {char }}>100 \mathrm{~m} \mathrm{~s}^{-1}$. As Fig. 10 (right column) shows, the number of distorted multiplets increases with increasing amplitude and these multiplets are located along straight lines in the $(l, v)$ diagram. The modes that drop out are those with the same penetration depth, i.e., they all have the same ratio $v / L$, indicating the same lower turning point. These modes therefore are the ones that will have the largest influence on the inferred rotation rate in this region. These modes probe the zones where the flow field is strongest.

For the rotation inversion the odd $a_{s}$ coefficients are used. The meridional flows and the rotation are orthogonal to each other; this is also mirrored in the splittings. As we have seen in Sect. 2, the expansion of the rotational splitting is given by the odd and the expansion of the meridional-flow-splitting is given by the even $a_{s}$ coefficients. We note that the calculated even $a_{s}$ coefficients are zero in the absence of meridional flows. As the orthogonality of the Wigner $3 \mathrm{j}$ symbols (Edmonds 1960) is given by

$$
\sum_{l_{3} m_{3}}\left(2 l_{3}+1\right)\left(\begin{array}{ccc}
l_{1} & l_{2} & l_{3} \\
m_{1} & m_{2} & m_{3}
\end{array}\right)\left(\begin{array}{ccc}
l_{1} & l_{2} & l_{3} \\
m_{1}^{\prime} & m_{2}^{\prime} & m_{3}
\end{array}\right)=\delta_{m_{1} m_{1}^{\prime}} \delta_{m_{2} m_{2}^{\prime}}
$$

and

$\sum_{m_{1} m_{2}}\left(\begin{array}{ccc}l_{1} & l_{2} & l_{3} \\ m_{1} & m_{2} & m_{3}\end{array}\right)\left(\begin{array}{ccc}l_{1} & l_{2} & l_{3}^{\prime} \\ m_{1} & m_{2} & m_{3}^{\prime}\end{array}\right)=\frac{\Delta\left(l_{1}, l_{2}, l_{3}\right)}{2 l_{3}+1} \delta_{l_{3} l_{3}} \delta_{m_{3} m_{3}^{\prime}}$,

where $\Delta\left(l_{1}, l_{2}, l_{3}\right)$ means that this factor is non-zero only if the conditions in Eq. (14) are satisfied. The Wigner $3 \mathrm{j}$ symbols $(s l l / 0 m-m)$ and $\left(s l l^{\prime} / 0 m-m^{\prime}\right)$, that correspond to rotational or meridional flows, respectively, are orthogonal with regard to $s$. That means that the meridional and the rotational flows can be 

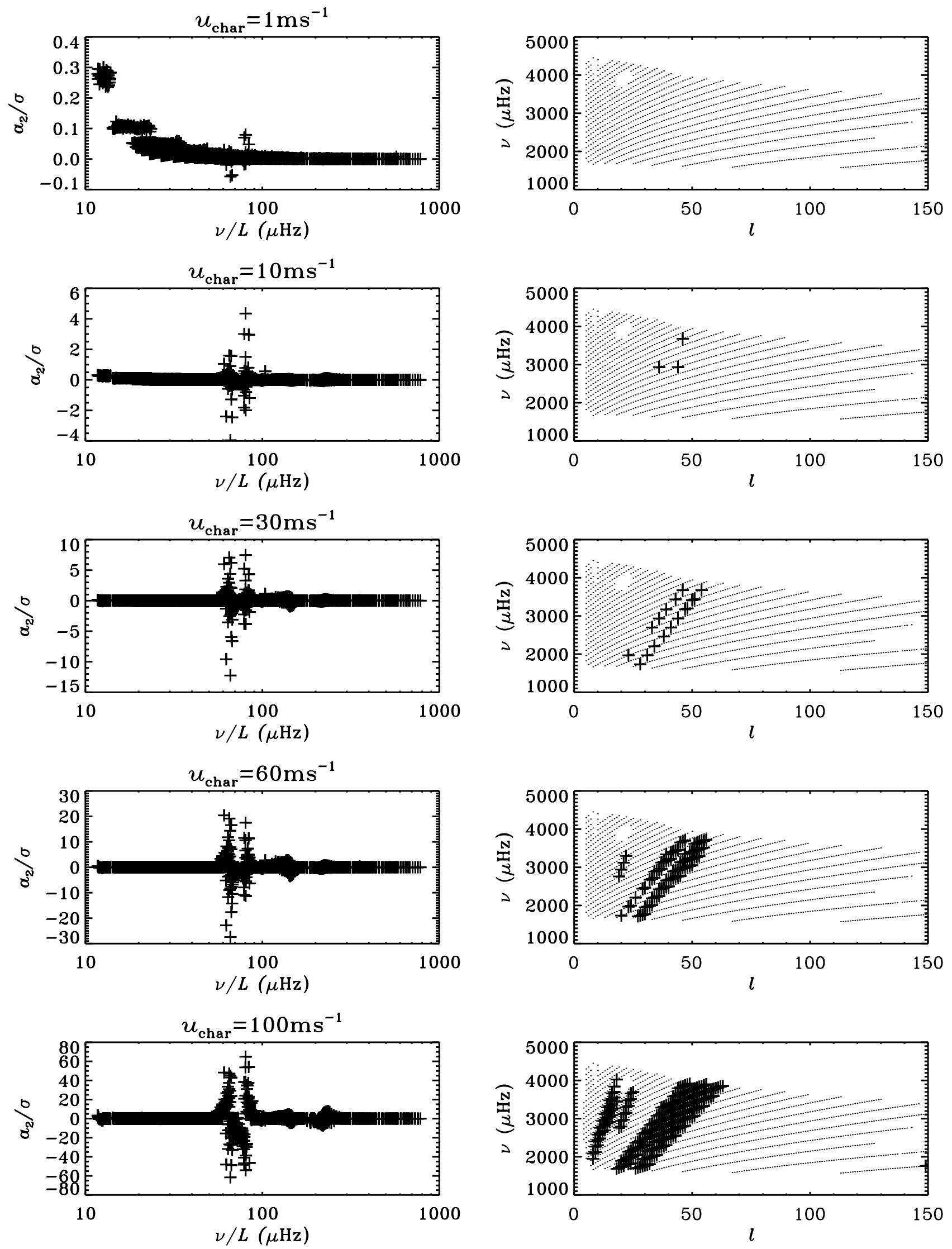

Fig. 10. Left: coefficients $a_{2}$ divided by the standard deviation of $a_{2}$. With increasing $u_{\text {char }}$ the influence on the coefficient becomes more visible as increasing deviation from zero. Right: $(l, v)$ diagrams. Bold symbols indicate multiplets where $a_{2}$ is more than three standard deviations away from zero. The number of distorted multiplets increases with increasing amplitude $u_{\text {char }}$. Both indicators show that the modes probing the region of the strongest velocity field (in this examples $s=8, t=0$ ) become affected. 
disentangled. Focusing on the even $a_{s}$-coefficients (Fig. 10 left column) the meridional flow becomes noticeable at an amplitude $u_{\text {char }}=10 \mathrm{~m} \mathrm{~s}^{-1}$.

Hence, even if the inversion of the odd $a_{s}$-coefficients itself does not show any changes in the rotation rate, because the rotation rate is not changed as there are no additional flows in the direction of the rotation, we can nevertheless detect the meridional flows, if their amplitude is larger than $10 \mathrm{~m} \mathrm{~s}^{-1}$. The new diagnostics are the distortion of the multiplets and the even $a_{s}$-coefficients.

\subsection{Model artifacts below the convection zone}

In this section we explain the emergence of artifacts below the convection zone for the poloidal flow components with $t \neq 0$. The main reason for this behavior is that we are inverting for differential rotation. However, the poloidal flow field components bring in additional flows in the direction of the rotation. Hence the odd $a_{s}$-coefficients and therefore the inferred rotation rate are changed due to crosstalk. This change in the coefficients does not vanish by the usage of averaging kernels in Eq. (26), because these kernels consists of eigenfunctions that do not necessarily couple by the poloidal flow.

As the Wigner $3 \mathrm{j}$ symbols $(\mathrm{sll} / 0 \mathrm{~m}-\mathrm{m})$ and $\left(\mathrm{sll} / \mathrm{tm}-\mathrm{m}^{\prime}\right)$ with $t \neq 0$ are not orthogonal, and as the basis of the functions used $(s l l / 0 m-m)$ is not complete, the two flows, differential rotation and poloidal field, cannot be disentangled. As a consequence, the total splittings in the multiplets are misinterpreted in terms of zonal toroidal flows. The inversion will then lead to a distorted differential rotation profile that is compatible with the distorted multiplets calculated from the combined effect of regular rotation and additional poloidal flow. For low amplitudes $u_{\text {char }}$ this is attained by extending the differential rotation deeper into the sun and changes in the rotation rate at low colatitudes, because the modes concentrated to the equator and penetrating deeply are affected most. For high amplitudes $u_{\text {char }}$ all modes are affected; the inversion results in a changed near surface rotation rate and in a strong latitudinal variation of the differential rotation, that is extended deep into the sun.

We conclude from our numerical experiment that pure meridional flows and pure sectoral cellular flows in certain convective cells can be detected with global helioseismic data. The detectability limit that we can derive on the basis of our models (Eqs. (2)-(9), (18), and (19)) of such large-scale flows is of the order of $|\boldsymbol{v}| \approx 10 \mathrm{~m} \mathrm{~s}^{-1}$.

\section{Discussion}

To determine the influence of large-scale flows on the inversion results, we use the observed average rotation rate as estimate of the true rotation rate. Observed mode splittings, derived from GONG or MDI time series, represent the solar rotation averaged over 108 days (shifted by 36 days) and 72 days respectively. As a consequence, the results of our numerical experiment represent large-scale flows with a life time of one or more solar rotations. Such a flow can have a maximum sectoral amplitude of about $10 \mathrm{~m} \mathrm{~s}^{-1}$. Otherwise, it will lead to a noticeable distortion of the rotation rate in the convection zone in contradiction to the observations. This conclusion relies on the observed smoothness of the $a$-coefficients and the inferred rotation rate. Any break in the rotation rate introduced by a large scale flow field would be noticeable. Since no such breaks are observed, the "true" solar rotation would have to precisely counteract the disrupting effect of the flow field which seems unlikely.

Zonal-meridional flows, as expected, have no direct influence on the rotation rate. However, a large zonal meridional flow can disrupt the structure of a multiplet and thus lead to a reduced coverage in the $(l, v)$-diagram. We find that a large-scale flow can have a maximum zonal amplitude of about $u_{\text {char }}=100 \mathrm{~m} \mathrm{~s}^{-1}$ without leading to noticeably reduced coverage. However, the even $a_{s}$-coefficients show the first indications of the flow field at about $u_{\text {char }}=10 \mathrm{~m} \mathrm{~s}^{-1}$. Thus our methods are similarly sensitive to flows in meridional and rotational direction.

Since the near-surface amplitude is much smaller than the maximum (cf., Fig. 6), this result is comparable to the most stringent constraint provided by previous surface observations which placed an upper limit of a few $\mathrm{m} \mathrm{s}^{-1}$ on the near-surface amplitude of giant cells (for example, LaBonte et al. 1981; Beck et al. 1998). Our results suggest that such long-lived large-scale structures do not exist in the convection zone of the Sun. This conclusion is supported by global simulations of the convection zone. Miesch et al. (2000) found that the convection structure is very time dependent in the turbulent case, making it difficult to identify any persistent features over the course of a full rotation. However, large-scale patterns with velocity amplitudes greater than $10 \mathrm{~m} \mathrm{~s}^{-1}$ might exist on time scales of days to a few weeks.

Long-lived large-scale patterns with amplitudes lower than $10 \mathrm{~m} \mathrm{~s}^{-1}$, which are not ruled out by our result, might allow speculations about the origin of the recently reported dynamic variations with a $1.3 \mathrm{yr}$ period at the base of the convection zone (Howe et al. 2000). There, the peak-to-peak variation of $\delta \Omega / 2 \pi$ in the rotation rate at $0.72 R$ is $6 \mathrm{nHz}$ which corresponds to a variation in the velocity of $\approx 9 \mathrm{~m} \mathrm{~s}^{-1}$. As this is of the order of the detectability limit of the inversions, we can speculate whether the physical background for these variations at the base of the convection zone are large-scale flows with a lifetime of $1.3 \mathrm{yr}$ that affected the inversions for rotation rate in the same manner as the model flows did in our experiment.

The final answer about the existence of large-scale flows on the basis of global helioseismology data cannot be given, as long as it is not possible to invert for such flows. The difficulty is that in general the flow component in longitude cannot be disentangled from the rotation. However, we are able to derive some restrictions on the existence of large-scale cells. Only the meridional flows can be clearly separated from the rotation. Therefore our geometry parameterization of the flows was adequate to derive an estimate of the detectability of various large-scale flows. But more complicated flows within a cell impose different maximum amplitudes in different directions. Hence new numerical experiments are necessary to give a more detailed insight into the detectability of the various flow components in the convection zone. For the work presented, the details of the geometry (number of cells) make no difference, 
but there may be more subtle effects which we will study in a later investigation. Then, in the near future, we might be able to answer the question, whether there exist large-scale flows in the convection zone, e.g., whether there exists a return flow of the meridional surface flow at the base of the convection zone, and whether this return flow can be detected with this method on the basis of global helioseismic data.

Acknowledgements. M. Roth thanks M. Stix for helpful discussion on and interest in this work. M. Roth thanks the NSO/GONG staff for hospitality during the visits in Tucson. This work was supported by DFG Grant STI 65/9 and by NASA Grant S-92698-F. This work utilizes data obtained by the Global Oscillation Network Group (GONG) project, managed by the National Solar Observatory, which is operated by AURA, Inc. under a cooperative agreement with the National Science Foundation. The data were acquired by instruments operated by the Big Bear Solar Observatory, High Altitude Observatory, Learmonth Solar Observatory, Udaipur Solar Observatory, Instituto de Astrofísico de Canarias, and Cerro Tololo Interamerican Observatory.

\section{References}

Backus, G. E., \& Gilbert, F. 1968, Geophys. J.R.A.S., 16, 169

Beck, J. G., Duvall, Jr. T. L., \& Scherrer, P. H. 1998, Nature, 394, 653

Bertello, L., \& Restaino, S. R. 1993, A\&A, 273, 260

Christensen-Dalsgaard, J., Däppen, W., Ajukov, S. V., et al. 1996, Science, 272, 1286

Christensen-Dalsgaard, J. 1998, Lecture Notes on Stellar Oscillations, Feb. 1998 http://www.ifa.au.dk/ jcd
Craig, I. J. D., \& Brown, J. C. 1986, Inverse problem in astronomy: a guide to inverse strategies for remotely sensed data (Adam Hilger, Bristol)

Cram, L. E., Durney, B. R., \& Guenther, D. B. 1983, ApJ, 267, 442.

Edmonds, A. R. 1960, Angular Momentum In Quantum Mechanics (Princeton University Press)

Elliott, J. R., Miesch, M. S., \& Toomre, J. 2000, ApJ, 533, 548

Haber, D. A., Hindman, B. W., Toomre, J., et al. 2000, Sol. Phys., 192, 335

Hathaway, D. 1992, Sol. Phys., 137, 15

Hathaway, D. H., Beck, J. G., Bogart, R. S., et al. 2000, Sol. Phys., 193, 299

Howe, R., Christensen-Dalsgaard, J., Hill, F., et al. 2000, Science, 287, 2456

LaBonte, B. J., Howard, R., \& Gilman, P. A. 1981, ApJ, 250, 796

Lavely, E. M., \& Ritzwoller, M. H. 1992, Phil. Trans. R. Soc. Lond. A, 339,431

Miesch, M. S., Elliott, J. R., Toomre, J., et al. 2000, ApJ, 532, 59

Ritzwoller, M. H., \& Lavely, E. M. 1991, ApJ, 369, 557

Robillot, J. M., Bocchia, R., Fossat, E., \& Grec, G. 1984, A\&A, 137, 43

Roth, M. 2001, ApJ, 559, 1165

Roth, M., \& Stix, M. 1999, A\&A, 351, 1133

Schröter, E. H., Wöhl, H., Soltau, D., \& Vázquez, M. 1978, Sol. Phys., 60, 181

Schou, J., Antia, H. M., Basu, S., et. al. 1998, ApJ, 505, 390

Simon, G. W., \& Weiss, N. O. 1968, Zs. Ap., 69, 435

Tikhonov, A. N., \& Arsenin, V. Y. 1977, Solutions of ill-posed problems (Winston, Washington DC)

Ulrich, R. 2001, ApJ, 560, 466 